\title{
General medical practitioners' knowledge and beliefs about osteoporosis and its investigation and management
}

Renée Otmar $^{1,2}$, Susanne D. Reventlow ${ }^{3}$, Geoffrey C. Nicholson ${ }^{4}$, Mark A. Kotowicz ${ }^{1}{ }^{2}$, Julie A. $\operatorname{Pasco}^{1,2}$

${ }^{1}$ School of Medicine, Deakin University, PO Box 281, Geelong, Victoria 3220, Australia

${ }^{2}$ Department of Medicine, Northwest Academic Centre, The University of Melbourne, Sunshine Hospital, 176 Furlong Road, St Albans, Victoria 3021, Australia

${ }^{3}$ The Research Unit for General Practice and Section of General Practice, Department of Public Health, Copenhagen University, Øster Farimagsgade 5, 1014 Copenhagen K, Denmark

${ }^{4}$ Rural Clinical School, School of Medicine, The University of Queensland, Private Mail Bag 2, Toowoomba, Queensland 4350, Australia

Corresponding author: Renée Otmar, Barwon Epidemiology and Biostatistics Unit, School of Medicine, Deakin University, PO Box 281, Geelong, VIC 3220, Australia; Tel +61 35226 7442; Fax: +6135246 5165; email rotmar@barwonhealth.org.au 


\section{Abstract}

Purpose We aimed to investigate barriers, enablers and other factors influencing the investigation and management of osteoporosis, using a qualitative approach. This paper analyses data from discussions with general medical practitioners (GPs) about their beliefs and attitudes regarding osteoporosis and its management.

Methods Fourteen GPs and two practice nurses aged 27-89 years participated in four focus groups, from June 2010 to March 2011. Each group comprised 3-5 participants, and discussions were semi-structured, according to the protocol developed for the main study. Discussion points ranged from the circumstances under which GPs would initiate investigation for osteoporosis and their subsequent actions, to their views about treatment efficacy and patient adherence to prescribed treatment. Audio recordings were transcribed and coded for analysis, using analytic comparison to identify the major themes.

Results The GPs were not particularly concerned about osteoporosis in their patients or the general population, ranking diabetes, osteoarthritis, cardiovascular disease and hypertension higher than concern about osteoporosis. They expressed confidence in the efficacy of antifracture medications, but were concerned about the potential financial burden on patients with limited incomes. The GPs were unsure about guidelines for investigation and management of osteoporosis in men, and the appropriate duration of treatment, particularly for the bisphosphonates in all patients.

Conclusions The GPs' ambivalence about osteoporosis appeared to stem from structural factors such as financial barriers for patients and their own beliefs about the salience of osteoporosis. GPs considered the impact of investigating and prescribing treatment in the context of patients' whole lives. 


\section{Mini abstract}

This qualitative study explored beliefs and attitudes regarding osteoporosis and its management.

General medical practitioners (GPs) were ambivalent about osteoporosis due to concern about financial barriers for patients and their own beliefs about the salience of osteoporosis. GPs considered investigation and treatment in the context of patients' whole lives.

Keywords osteoporosis * qualitative methods * general medical practitioners 


\section{Introduction}

Osteoporosis and associated fractures represent a substantial burden of disease worldwide [1]. Although there is good evidence for the efficacy of a range of pharmacological interventions to prevent fracture, reduce bone loss and/or improve bone mineral density [2-4], treatment adherence rates are suboptimal: a 2007 review of 13 international observational studies found treatment adherence rates in the range 10-68 per cent [5]. It has been suggested that medical practitioners do not initiate investigation or treatment for osteoporosis because, among other reasons, they do not understand or follow guidelines for primary care of osteoporosis and consider the costs to patients to be prohibitive [6-9]. However, insufficient evidence exists to enable appropriate intervention at both clinical and public health levels.

The main study aimed to investigate barriers, enablers and other factors influencing the investigation and management of osteoporosis, from the perspectives of consumers and medical practitioners. This paper analyses data obtained from focus group discussions with general medical practitioners (GPs), in which we sought to uncover the GPs' beliefs and attitudes regarding osteoporosis and its management, including their views on the efficacy of osteoporosis and anti-fracture medications.

\section{Defining osteoporosis}

It may be assumed that medical practitioners share a single definition of osteoporosis - by virtue of their training and expectations that they maintain knowledge of current research - but this is not necessarily the case. Over the past two centuries there has been a shift in the medical understanding of osteoporosis, from its 19th-century portrayal of a deteriorated, porous human bone and a focus in the 20th century on prevalence of fracture due to hormonal changes in menopausal women, to the present characterisation of osteoporosis as a disease to be treated or cured and/or as a manageable risk factor for fracture [10-12]. Attempts to define osteoporosis in 
terms of a threshold value for bone mineral density (BMD) have contributed towards this ambiguity. One view defines osteoporosis by degree of severity, so that 'established osteoporosis' is defined by low BMD measures coupled with fracture, and 'osteopenia' means a low BMD measure with no history of fracture $[13,14]$. Another considers BMD to be the risk factor, while osteoporosis is the 'disease', characterised, in part, by reduced BMD. As observed by Skolbekken and colleagues [12], evolution in the understanding of osteoporosis has shifted attention 'from those who experience fractures to those at risk of doing so'.

\section{A focus on prevention}

Coupled with this shift in the medical understanding of osteoporosis has been a call, and increasingly an expectation in the public health sector, for preventative measures in general practice as well as specifically related to chronic disease - osteoporosis included. This is despite 'serious deficits' being reported in health professionals' knowledge of the prevention and management of osteoporosis [15]. Thus, in contemporary medical practice expectations of GPs are heightened and increasingly present professional, medico-legal and other imperatives for GPs to transform the well-worn, 15-minute medical consultation from treating the sick to treating the potentially sick. The question of how GPs respond to these demands for a preventative approach in their practice underpins the analysis presented in this paper. Surveys of medical practitioners have reported that physicians have long perceived osteoporosis to be a normal aspect of ageing [16], and that up to 38 per cent of GPs under-estimated the prevalence of osteoporosis [17]. We hypothesised that the views of GPs in our study would be consistent with the latter two findings, and that GPs' practices in investigating and managing osteoporosis would be similarly consistent with a perception of osteoporosis as having low salience.

\section{Materials and methods}

\section{Setting and recruitment}

In Australia, dual energy x-ray absorptiometry (DXA) and osteoporosis therapy for approved indications are subsidised by a national, taxpayer-funded, health insurance scheme (Medicare). 
DXA is available for evaluation of low-trauma fracture, monitoring in individuals with low BMD, fracture risk assessment in those aged 70 years and older and evaluation of individuals with diseases or exposures known to reduce BMD or predispose to fracture. The out-of-pocket cost to patients who do not meet these criteria is in the region of $\$ 100$ (AUD). Osteoporosis therapy is subsidised under the national Pharmaceutical Benefits Scheme (PBS) for secondary fracture prevention and for primary prevention of fracture in those aged 70 years and older with a BMD T-score below minus 2.5 standard deviations of the mean for young adults of the same sex and in patients on chronic glucocorticoid therapy.

In Australia, a consultation with a GP is paid for by Medicare for eligible cardholders. Some GPs 'bulk-bill' Medicare for all or some of their patients; these patients do not have to pay for the consultation, providing they can present their Medicare card. Other patients are required to pay the GP's full fee and then may claim a refund from Medicare for the eligible portion of the fee.

The study was undertaken in Geelong, a regional city in south-eastern Australia, which has a moderately large, stable population (160,991 at the 2006 Census) [18]. According to the General Practitioners Association of Geelong (GPAG), 270 GPs were practising in the region in early 2011 - some 19 in sole practice and 47 in practices shared between two or more GPs (personal communication, 17/01/2011).

All participants were recruited in the context of general medical practice in the study region. Normally, practices in the study region do not identify as having specialty expertise or interests, and participants were not asked about whether they had a special interest in osteoporosis. At the time of the study no Fracture Liaison Service existed in the region. 
We invited GPs practising in the region to participate in the study through focus groups. Focus groups provide an efficient method by which to canvass the views of participants through a group process that allows for exploration of a topic in greater depth than possible through interview [19]. Recruitment was by two methods. Participants in the first focus group were recruited from registrants to an event hosted by GPAG. This educational event, organised exclusively for GPAG's members, included a guest speaker on osteoporosis (a prominent international researcher in the field). The focus group discussion was held prior to the guest speaker's presentation in order ensure that the GPs presented their own views and attitudes, rather than views influenced by the guest speaker's presentation. Continuing Medical Education (CME) points were accruable to GPs who attended this event. Subsequent focus groups were arranged directly with the medical practices that volunteered to participate, using a convenience and snowballing method of recruitment. We identified medical practices that would be ideal for inclusion - representing, for example, a client base of primarily older people, a rural or urban location, or an age or gender-based mixture of GPs working together in a shared practice. We were particularly interested in consulting with GPs working in a shared environment, given that the majority of medical practices in Geelong are run on this basis.

The convenience sampling method supported the development of a coding structure so that recruitment would be finalised at the point of theoretical saturation, when no further or new concepts were emerging during the focus group discussions [20]. The focus groups were conducted over a six-month period in 2010-11.

\section{Ethics}

The study was conducted in accordance with the World Medical Association Declaration of Helsinki [21]. The Barwon Health Human Research Ethics Committee provided full approval for the study, including the participant information and consent form (PICF), a one-page 
demographics questionnaire and the discussion protocol used to facilitate the focus group discussions. All participants read and signed a PICF prior to their participation.

\section{Data gathering and analysis}

Four focus groups were held in 2010-11, each comprising 3-5 participants and of 60-90 minutes' duration. The focus group discussions were semi-structured, according to the discussion protocol developed in the design of the main study. Discussion points ranged from the circumstances under which the GPs would initiate an investigation for osteoporosis and their subsequent actions, to their views about treatment efficacy and patient adherence to prescribed treatment.

The data comprised audio recordings and field notes of the focus group discussions. We used the 'analytic comparison', or 'nominal comparison', method derived from the work of 19th-century British philosopher and social thinker John Stuart Mill [22]. Analytic comparison is particularly useful in comparing data taken from a small number of cases. The data are organised into discrete categories, for each of which a concise explanation is put forward. Next, these categories are mined for any known or new patterns suggesting regularity within a specific social context, and contrasted with additional and alternative explanations [22]. Thus, the analytic comparison that follows seeks to identify patterns among GPs who were practising within the Geelong region, rather than seeking patterns that might apply (for example) to GPs practising across Australia, or in other parts of the world. This method is similar to the systematic approach promoted by Bradley and colleagues [20], which integrates both inductive and deductive processes. Given that the major themes identified for discussion in the focus groups were largely predetermined, and integrated 'concepts already well known in the extant literature' [20], much of our analysis is by definition deductive. However, applying a method of constant comparison $[23,24]$ during line-by-line reviewing and coding of the data allowed codes to be assigned to 
reflect new concepts emerging from the data, and thus to remain open to new interpretations of the topics discussed.

The first author (RO) coded the data and developed the initial coding schemes, which were reviewed by the co-authors. The audio recordings of the focus group discussions were transcribed using commercially available word-processing software, and then imported into NVivo9 (QSR International, 2011) for computer-aided qualitative data analysis. The process involved thematic coding according to the overarching themes of interest in the present analysis, and initially this generated several dozen nodes that were pruned through systematic merging, renaming and reorganising. The remaining nodes were subsequently coded into categories and subcategories as described in the results section.

\section{Participants}

The GPs in the four focus groups comprised three females and 11 males, ranging in age from 27 to 89 years. There were also two female practice nurses, aged 41 and 52 years, respectively.

The practice nurses were unintended participants in the study; they were invited by the GPs in the large, mixed medical practice in which they worked to participate in the focus group discussion, as it was felt that practice nurses played an important role in that particular medical practice. It should be noted that the data were analysed with and without the views of the practice nurses, as a check for contextual sensitivity,[25] and the results were essentially identical. This was not surprising, given that the practice nurses were employed in the medical practice in which the focus group was conducted and took direction from the GPs who employed them.

\section{Results}


Although they agreed that it was potentially a debilitating condition, osteoporosis was something the GPs in our study were not particularly concerned about, regarding their patients or the population as a whole. They ranked their own concerns about adults living with diabetes, osteoarthritis, cardiovascular disease, hypertension, cancers and depression higher than concern about osteoporosis. In terms of the general population, infectious diseases that have an impact on infant mortality were of greater concern than osteoporosis.

Few participants said that they would initiate an investigation for osteoporosis as a preventative measure; most did so only when certain risk factors presented in the course of a consultation with the patient. In considering the investigation and management of osteoporosis, all participants focused their attention on postmenopausal and elderly women, for the most part to the exclusion of men and younger women. Indeed, the minority of GPs who did discuss the management of osteoporosis in men were unsure about the guidelines and the available evidence to support their work with men. Primary prevention in women younger than 65 years was relegated to a utopian healthcare system - an ideal world in which sufficient time, resources and motivation were available.

All participants expressed confidence in the efficacy of osteoporosis/anti-fracture medications available on the market. However, several were concerned that the costs of these medications would be prohibitive for many of their patients. While this concern reflected the GPs' consideration of their patients' whole lives in context, it also affected their management of the condition by pharmacological means.

\section{GPs' knowledge, beliefs and attitudes about osteoporosis}

In this section we report on the GPs' knowledge, beliefs and attitudes regarding osteoporosis, including its investigation and management, and the efficacy of available treatments. For the 
analytic comparison, the data were organised into the following discrete categories, based on the flow of the discussions within the groups, the intensity of interest displayed by participants during the discussions and any unexpected topics that arose. The category titles selected are selfexplanatory and are linked directly with the focus of this paper and the aims of the broader study:

- Approach to osteoporosis investigation and diagnosis

- Approach to osteoporosis management/treatment

- Beliefs about osteoporosis treatments and their efficacy

\section{Approach to osteoporosis investigation and diagnosis}

At the start of each focus group discussion we asked the question 'Under what circumstances would you investigate - or consider investigating - for osteoporosis?' The GPs' responses were not unexpected, given that there are established guidelines for doing so [26], and for the most part their views were not controversial. The GPs reported that they investigated for osteoporosis when an older patient presented with a fragility (or 'low-trauma') fracture, or when a patient reported or was known to have been taking certain prescribed medications, such as glucocorticoids and anticonvulsants, for an extended period of time.

Three GPs said they automatically would commence investigation of a woman's BMD when she reached menopausal age - around 50 years - and that this might be one of a raft of investigations (for example, to check cholesterol, vitamin D levels etc.) initiated to assist in monitoring their patients' health as they entered middle age. These early investigations formed the baseline measures for future monitoring. But this concept of a 'standard' screening approach was controversial among some GPs, who felt either that this was an unnecessary and possibly a wasteful use of limited resources, or that the 'system' (that is, the guidelines and accepted indications for reimbursement for investigation and treatment of osteoporosis) restricted them from taking what may be perceived as the ideal approach to prevention or early intervention. The 
GPs who gave their perimenopausal and early postmenopausal patients a thorough baseline health check took what they described as an holistic approach to their perceived duty of care. Others commenced investigation for osteoporosis only upon presentation of known risk factors or otherwise strong evidence of bone deterioration (such as a report from a specialist). A complicating factor was that among this latter group were GPs who were concerned that time and cost restrictions limited their ability to take a preventative approach, which could mean (for example) regularly screening all women from, say, age 50 years, for bone density, calcium and vitamin D levels.

In discussing osteoporosis, the GPs concentrated their attention on the treatment and prevention of osteoporosis in women, and most particularly in postmenopausal and older women. They believed that osteoporosis was a relatively uncommon occurrence in men, and there was a sense of confusion about what might be appropriate trigger points for investigation in men, apart from guidelines relating to risk factors in men aged 70 years and older. One (female) GP explained this approach and her reasons for not including men in regular screening for osteoporosis. She also suggested that she might ask a visiting endocrinologist for advice on the matter:

You know, I don't screen men ... men's a big question mark for me. See, I mean, it's obviously not rebatable for them. I don't know whether it is in the over 70s [age group]. That's something I could ask [endocrinologist], whether it is. [Men] tend to get it later, I guess. They haven't been focused on because everyone's living longer now. (Female GP, 48 years)

The GPs' investigations for osteoporosis involved sending their patients for a BMD scan, which in the Geelong region is undertaken using the DXA method, considered to be the most consistent and reliable measure of BMD [27]. Some GPs said the cost of DXA prohibited them from discretionary screening for osteoporosis in people they felt were at risk of fracture, if they thought that the patient would not be able to afford it.

The DXA report sent to the GP following a patient's BMD scan includes both the T-score, which compares the patient's BMD with that of a normal young reference, and the Z-score, which 
compares the patient's BMD with that of peers of the same age $[28,29]$; the latter being commonly reported by the GPs in the present study as being a useful tool in explaining osteoporosis to their patients and in prescribing treatment. In fact, the DXA report seemed to instil in the GPs a high degree of confidence in diagnosing, monitoring and managing osteoporosis.

One GP questioned the value of using BMD in diagnosing osteoporosis. She was of the view that questions remained in the field regarding whether BMD was an appropriate measure of bone strength.

\section{Approach to osteoporosis management/treatment}

In patients with a diagnosis of established osteoporosis (T-score $\leq$ minus 2.5 with/without prior fracture), all of the GPs said or implied that they followed the RACGP clinical guidelines for treatment [30]. In patients with osteopenia or bone deficits, the GPs' approach to management differed somewhat, and they tended to fall into two camps: One offered lifestyle interventions involving changes in diet and exercise as well as calcium and/or vitamin D supplementation, as a first-line approach. This was favoured by GPs who generally were positive in their views about their patients, particularly patients' ability to understand what was needed from them, and about patients' commitment to making the necessary lifestyle changes. If the patient was unable (that is, not unwilling) to adhere to the prescribed program of lifestyle change, these GPs prescribed osteoporosis medication as a next step (though not necessarily as a last resort). GPs in the other camp believed that prescribing the appropriate medication immediately following a diagnosis of reduced bone mass (regardless of severity) was the best chance for patients to gain an improvement in their BMD and to prevent fracture. These GPs said they lacked confidence in their patients' ability and commitment to lifestyle change.

Some GPs expressed concern about the cost of osteoporosis medications, which they felt was 
prohibitive for some patients, particularly those living on a limited or fixed income, such as the government age pension, and who were not eligible for subsidised medication under the PBS.

This included early retirees who did not have a history of fracture.

One GP said that she did not know how long to maintain her patients safely on osteoporosis medications, particularly bisphosphonates. This issue was not raised in any of the other focus groups, nor did other members of her focus group respond to the GP's statement.

\section{Beliefs about osteoporosis medications and their efficacy}

The GPs expressed a high level of confidence in the efficacy of the osteoporosis medications they were prescribing, as well as the available evidence from research. They relied upon information from three sources: the advice of well-known specialists in the region who had strong credentials in osteoporosis research; published research in the major peer-reviewed medical journals; and their own experience in monitoring their patients over time. They acknowledged that some people experienced side effects in taking certain osteoporosis medications, such as oral bisphosphonates, but were confident that sufficient alternatives were available on the pharmaceuticals market to meet their needs.

One GP questioned whether treating elderly patients for osteoporosis following fracture was a cost-effective approach from a public health perspective. An older, male GP in the same focus group agreed with him, but overall this view stood out from those of the rest of the participants:

I sometimes wonder about the cost benefit. You know, in terms of how many fractures are we really saving? How many people do we have to treat to save, you know, a fracture? And what group of people are we preventing the fractures in? You know, if we were preventing the fractures in the fifty to sixty year old, I'd say that's a bloody good thing, but most of these fractures occur in people who are at the end of their lives and the sort of fractures that are more common, like the vertebral fractures, well, people don't die from that. (Male GP, 57 years)

Although his was a reasoned and considered opinion, it should be noted that the same GP was generally pessimistic about patients and osteoporosis treatments. He felt that his role in 
preventing chronic disease was undermined by a lack of commitment in the general community towards healthy lifestyles. 


\section{Discussion}

The analytic comparison of the data suggests several key themes or trends in the GPs' knowledge, beliefs and attitudes regarding osteoporosis, its investigation and management.

The data reveal a sense of ambivalence in the GPs' beliefs and attitudes about osteoporosis. They were unanimous in their belief that osteoporosis was potentially a debilitating condition, particularly in old age, but any concern they felt about it was outweighed by the demands of health conditions perceived as having higher salience to them and their patients, such as diabetes and cardiovascular disease. It may be that this reflects the GPs' response as a profession to demands that they shift their attention 'from the sick to the potentially sick' [31], as discussed in the introduction to this paper. It may also signal the GPs' attention to individual patient needs, in the context of the patient's overall health and life circumstances. For example, in a patient who has a more urgent or life-threatening condition that requires careful management of medication, osteoporosis medication might not be a feasible addition.

There were many reasons the GPs did not consistently investigate for osteoporosis, though when they did so they recognised that effective investigative technologies and treatment options were available. The GPs' ambivalence appears to stem from structural factors, such as patient financial barriers (real or perceived), as well as the GPs' own beliefs about the salience of osteoporosis, among themselves and their patients.

The GPs suggested that a standardised, baseline, general health assessment would be ideal for all people as they entered middle age. However, only a small minority of the GPs in the study undertook this type of screening among their patients. Among the reasons for not doing so was a perception that cost would be a barrier for many patients who would be unable to afford DXA scanning and other tests for non-essential or non-acute investigation, and time constraints. Here, again, the GPs expressed ambivalence about their role in prevention of fracture. That their 
unease about initiating an investigation centred on their concern about costs to patients might be challenged by their simultaneous views that screening itself may be a waste of resources. The views of the GP who questioned the value of using BMD in diagnosing osteoporosis are reflected in the literature on measurement of BMD [see, for example, 27, 32]. The other GPs in the study did not quote the guidelines or the literature when discussing their use of DXA, but relied upon their own experience to guide their practice.

Another pertinent factor is that little is known about how GPs use DXA scanning in the investigation and treatment of osteoporosis [8, 33]. A study in the United Kingdom exploring GPs' beliefs about diagnosis and management of osteoporosis, including the role of DXA scanning, reported that GPs lacked confidence in using DXA scanning as a diagnostic tool, largely due to misperceptions about how to use the local service [8]. This finding is in contrast to our study, where the GPs expressed a high level of confidence in DXA scanning, both as a diagnostic and a monitoring/management tool for osteoporosis. This may be because, as one GP put it, the Geelong region has been fortunate to have the support of a medical department (at Barwon Health) with a strong interest, expertise and international research reputation in the field of bone health. It is thus reasonable to assume that the use of DXA scanning has featured in the cultural transmission of information about osteoporosis investigation within the medical profession in the region [34].

It should be noted here, however, that little evidence is available to support mass screening for osteoporosis in people younger than 65 years of age and in the absence of other clinical risk factors [35], since poor adherence to drug therapies reduces the cost effectiveness of screening [36]. Furthermore, re-screening may be required less frequently than previously thought, as reported by a recent follow-up in the longest-running osteoporosis study in the United States [37]. The medical literature also indicates that mass screening for chronic health conditions such 
as osteoporosis may be beyond the capacity of healthcare systems, even in relatively wealthy nations. For example, Petursson and colleagues [38] observed that the potential workload associated with guidelines for the management of hypertension in Europe 'could destabilise the healthcare system in Norway, one of the world's most long- and healthy-living nations'.

The GPs' beliefs about the efficacy of osteoporosis medications gave them confidence in their treatment prescriptions, and might be considered a strong enabler in the management of osteoporosis. However, this confidence was undermined by external factors, such as adverse media reports regarding osteoporosis medications. A recent study investigating the impact of a national current affairs television program, linking osteonecrosis of the jaw with bisphosphonate use, found a reduction in subsequent prescription use, such that 70 hip fractures, 60 other fractures and 14 deaths could have been prevented over the nine months of the study [39]. This vindicates our participants' concerns about the influence of such external factors on their management of osteoporosis.

Some GPs felt there were gaps in their knowledge about osteoporosis treatment, such as how long to maintain patients safely on certain medications and what is appropriate in calcium and vitamin D supplementation. Their concerns are not unfounded, given the recent controversies in the literature arising from reports of increased risk of cardiovascular events in patients on calcium supplementation [40], and increased risk of falls and fractures in patients on vitamin D supplementation [41]. The GPs' confusion regarding the evidence and guidelines in relation to these aspects of osteoporosis management is therefore not surprising.

The GP who questioned the cost-effectiveness of treating elderly patients for osteoporosis demonstrated a lack of familiarity with current evidence regarding the numbers needed to treat (NNT) [42-44]. There is evidence that fracture risk can be mitigated with pharmacological 
intervention in patients over age 50 with fracture [45]. The latent ageism underpinning this GP's view also raises questions about the perception of elderly patients by GPs, and their beliefs about the appropriate age at which to commence and/or cease treatment for osteoporosis.

The sharing of expertise, new ideas and the latest research was a strong feature of the shared medical practices that participated in the study. Theories of learning and cultural transmission suggest that formal education, such as the GPs' medical training and continuing medical education, serve as 'powerful mechanisms of cultural transmission' [34]. GPs working on their own may be just as likely to be exposed to the latest developments in medical research as their colleagues in shared medical practices, but changes in day-to-day practice are likely to be consolidated through the cultural transmission that naturally takes place in a shared medical practice.

While the present analysis provides a broad overview of GPs' beliefs and attitudes regarding osteoporosis investigation and treatment in the Geelong region and this presents a significant strength of the study, the nature of the study is such that its findings cannot be generalised to the broader Australian population. The focus groups in our study were limited by the participation of medical practices that expressed an interest in participating and, presumably, were interested in a discussion about osteoporosis. Needless to say, consultations with different groups and perhaps even with individual GPs may have produced different findings. The availability of osteoporosis expertise within the region is also not typical of all regions in the country.

Our hypothesis that the views of GPs in our study would be consistent with findings from other studies, that GPs perceived osteoporosis as a normal aspect of ageing and under-estimated its prevalence, was not supported. However, the hypothesis that GPs' practices in investigating and managing osteoporosis would be similarly consistent with a perception of osteoporosis as having 
low salience was supported. This may explain the low level of initiation of osteoporosis investigation for primary and secondary preventative purposes among our participants. It is consistent with a recent survey of Dutch general practitioners, which found that GPs tended to perform preventive measures for a range of health disorders (such as cardiovascular disease and kidney disease) only when patients asked for it during a consultation. In fact, only a quarter of the GPs surveyed in that study actively initiated consultations for preventative purposes [46].

\section{Conclusion}

This paper has analysed focus group data to illuminate GPs' beliefs and attitudes regarding their investigation and management of osteoporosis, including the efficacy of osteoporosis and antifracture medications. It demonstrates that multiple barriers to investigation, treatment and prevention of osteoporosis are likewise matched by enablers. Structural factors such as patient ability to pay for DXA scanning and/or osteoporosis medications should be considered as important as the GPs' own knowledge, beliefs and attitudes towards osteoporosis investigation, management and prevention.

Through the analysis presented here, we gained insights into why many GPs did not consistently and broadly initiate discussion, investigation and subsequent management of osteoporosis among patients who may have been at risk of fragility fracture: because there were self-reported gaps in their knowledge of osteoporosis medications and because they considered the impact of prescribing treatment in the context of patients' whole lives, including their ability to pay for those medications.

Acknowledgements: No disclosures. 


\section{References}

1. Johnell O, Kanis JA (2006) An estimate of the worldwide prevalence and disability associated with osteoporotic fractures. Osteoporosis International 17:1726-1733

2. Alonso-Coello P (2009) Is too much intervention recommended in the ACP osteoporosis treatment guidelines? In López A, Pencille LJ, Montori VM (eds) Annals of Internal Medicine. American College of Physicians, pp 285-287

3. Lock CA, Lecouturier J, Mason JM, Dickinson HO (2005) Lifestyle interventions to prevent osteoporotic fractures: A systematic review. Osteoporosis International 17:20-28 4. Sanders KM, Nicholson GC, Watts JJ, Pasco JA, Henry MJ, Kotowicz MA, Seeman E (2006) Half the burden of fragility fractures in the community occur in women without osteoporosis. When is fracture prevention cost-effective? Bone 38:694-700

5. Papaioannou A, Kennedy CC, Dolovich L, Lau E, Adachi JD (2007) Patient adherence to osteoporosis medications: Problems, consequences and management strategies. Drugs \& Aging 24:37-55

6. Chiang A, Jones J, Humphreys J, Martin C (2006) Osteoporosis: Diagnosis and treatment in a general practice population. Australian family physician 35:166-168

7. Inderjeeth CA, Glennon D, Petta A (2006) Study of osteoporosis awareness, investigation and treatment of patients discharged from a tertiary public teaching hospital. Internal Medicine Journal 36:547-551

8. Richardson JC, Hassell AB, Thomas E, Hay EM (2004) GPs' perceptions of the role of DEXA scanning: An exploratory study. Family practice 21:51-53

9. Rizzoli R, Brandi ML, Dreinhofer K, Thomas T, Wahl DA, Cooper C (2010) The gaps between patient and physician understanding of the emotional and physical impact of osteoporosis. Archives of Osteoporosis 5:145-153

10. Reventlow S, Bang H (2006) Brittle bones: Ageing or threat of disease-Exploring women's cultural models of osteoporosis. Scandinavian Journal of Public Health 34:320-326

11. Reventlow S, Overgaard I, Hvas L, Malterud K (2008) Metaphorical mediation in women's perceptions of risk related to osteoporosis: A qualitative interview study. Health, Risk \& Society 10:103-115

12. Skolbekken J-A, Østerlie W, Forsmo S (2008) Brittle bones, pain and fractures - Lay constructions of osteoporosis among Norwegian women attending the Nord-Trøndelag Health Study (HUNT). Social Science \& Medicine 66:2562-2572

13. Kanis J, Brazier JE, Stevenson M, Calvert NW, Lloyd Jones M (2002) Treatment of established osteoporosis: A systematic review and cost-utility analysis. Health Technology Assessment 6:1-146

14. Kanis JA, Burlet N, Cooper C, Delmas PD, Reginster J-Y, Borgstrom F, Rizzoli R (2008) European guidance for the diagnosis and management of osteoporosis in postmenopausal women. Osteoporosis International 19:399-428

15. Werner P (2005) Knowledge about osteoporosis: Assessment, correlates and outcomes. Osteoporosis International 16:115-127

16. Edwards L, Fraser M (1997) How do we increase awareness of osteoporosis? Ballieres Clinical Rheumatology 11:631-644

17. Werner P, Vered I (2002) The diagnosis of osteoporosis: Attitudes and knowledge of Israeli physicians. Aging: Clinical and Experimental Research 14:52-59

18. Australian Bureau of Statistics (2007) 2006 Census of Population and Housing. In. Commonwealth of Australia, Canberra

19. Kitzinger J (2006) Focus groups. In Pope C, Mays N (eds) Qualitative research in health care, 3 edn. Blackwell Publishing, Oxford, UK

20. Bradley EH, Curry LA, Devers KJ (2007) Qualitative data analysis for health services research: Developing taxonomy, themes, and theory. Health Services Research 42:1758-1772 
21. World Medical Association (2008) World Medical Association Declaration of Helsinki: Ethical principles for medical research involving human subjects. In, 59th General Assembly, Seoul, Korea edn.

22. Neuman WL (2003) Social research methods: Qualitative and quantitative methods. Allyn and Bacon, Boston, USA

23. Glaser BG, Strauss AL (1967) The discovery of grounded theory: Strategies for qualitative research. Aldine, Chicago, USA

24. Boeije H (2002) A purposeful approach to the constant comparative method in the analysis of qualitative interviews. Quality and Quantity 26:391-409

25. Silverman D, Marvasti A (2008) Doing qualitative research: A comprehensive guide. Sage Publications, Thousand Oaks, CA

26. O'Neill S, MacLennan A, Bass S, et al. (2004) Clinical practice: Guidelines for the management of postmenopausal osteoporosis for GPs. Australian family physician 33:910-917

27. Sturtridge W, Lentle B, Hanley DA (1996) Prevention and management of osteoporosis: Consensus statements from the Scientific Advisory Board of the Osteoporosis Society of Canada. 2. The use of bone density measurement in the diagnosis and management of osteoporosis. Canadian Medical Association journal 155:924-929

28. Henry MJ, Pasco JA, Nicholson GC, Pocock NA, Kotowicz MA (2004) Reference ranges for bone densitometers adopted Australia-wide: Geelong Osteoporosis Study. Australas Radiology 48:473-475

29. Henry MJ, Pasco JA, Korn S, Gibson JE, Kotowicz MA, Nicholson GC (2010) Bone mineral density reference ranges for Australian men: Geelong Osteoporosis Study. Osteoporosis International 21:909-917

30. The Royal Australian College of General Practitioners R (2010) Clinical guideline for the prevention and treatment of osteoporosis in postmenopausal women and older men. In. RACGP, South Melbourne, Vic., Australia

31. Skolbekken JA (1995) The risk epidemic in medical journals. Social Science \& Medicine 40:291-305

32. Antonacci MD, Hanson DS, Heggeness MH (1996) Pitfalls in the measurement of bone mineral density by dual energy x-ray absorptiometry. Spine 21:87-91

33. Walker-Bone K, Reid D, Cooper C (1998) Is screening for osteoporosis worthwhile? British Medical Bulletin 54:915-927

34. Hruschka DJ, Hadley C (2008) A glossary of culture in epidemiology. Journal of Epidemiology and Community Health 62:947-951

35. Gourlay M (2009) Osteoporosis screening: Mixed messages in primary care. American Family Physician 79:189-190

36. Hiligsmann M, Gathon HJ, Bruyère O, Ethgen O, Rabenda V, Reginster JY (2010) Costeffectiveness of osteoporosis screening followed by treatment: The impact of medication adherence. Value in Health: The Journal Of The International Society For Pharmacoeconomics And Outcomes Research 13:394-401

37. Gourlay ML, Fine JP, Preisser JS, May RC, Li C, Lui L-Y, Ransohoff DF, Cauley JA, Ensrud KE (2012) Bone-density testing interval and transition to osteoporosis in older women. The New England journal of medicine 366:225-233

38. Petursson H, Getz L, Sigurdsson JA, Heltlevik I (2009) Current European guidelines for management of arterial hypertension: Are they adequate for use in primary care? Modelling study based on the Norwegian HUNT 2 population. BMC Family Practice 10:

39. Sambrook PN, Chen JS, Simpson JM, March LM (2010) Impact of adverse news media on prescriptions for osteoporosis: Effect on fractures and mortality. Medical Journal of Australia 193:154-156

40. Bolland MJ, Barber PA, Doughty RN, Mason B, Horne A, Ames R, Gamble GD, Grey A, Reid IR (2008) Vascular events in healthy older women receiving calcium supplementation: Randomized controlled trial. British Medical Journal 336:262-266 
41. Sanders KM, Stuart AL, Williamson EJ, Simpson JA, Kotowicz MA, Young D, Nicholson GC (2010) Annual high-dose oral vitamin D for falls and fractures in elderly women: A randomised controlled trial. Journal of the American Medical Association 303:1815-1822 42. Reginster J-Y (2011) Antifracture efficacy of currently available therapies for postmenopausal osteoporosis. Drugs 71:65-78

43. Schwarz P, Jorgensen NR, Mosekilde L, Vestergaard P (2011) The evidence for efficacy of osteoporosis treatment in men with primary osteoporosis: A systematic review and metaanalysis of antiresorptive and anabolic treatment in men. Journal of Osteoporosis 2011:259818 44. Ringe JD, Doherty JG (2010) Absolute risk reduction in osteoporosis: Assessing treatment efficacy by number needed to treat. Rheumatology International 30:863-869

45. Åstrand J, Thorngren K-G, Tägil M, Åkesson K (2008) 3-year follow-up of 215 fracture patients from a prospective and consecutive osteoporosis screening program. Fracture patients care! Acta Orthopaedica 79:404-409

46. Nielen MM, Assendelft WJ, Drenthen AJM, van den Hombergh P, van Dis I, Schellevis FG (2010) Primary prevention of cardio-metabolic diseases in general practice: A Dutch survey of attitudes and working methods of general practitioners. European Journal of General Practice $16: 139-142$ 